\title{
Comparative Simulation Study Of LEACH-Like And HEED-Like Protocols Deployed In Wireless Sensor Networks
}

\author{
Soumia Laroui andMohammed Omari \\ LDDI Laboratory, Mathemetics and Computer Science Department, University of Adrar, Adrar 01000, Algeria
}

\begin{abstract}
WSNs represents one of the most interesting research areas with deep impact on technological development because of their potential usage in a wide variety of applications such as fire monitoring, border surveillance medical care, and highway traffic coordination. Therefore, WSNs researchers have defined many routing protocols for this type of network. In this paper, we have implemented and analyzed different clustering protocols, namely LEACH, LEACH-C, LEACH-1R, and HEED using MATLAB environment. These routing protocols are compared in different terms such as residual energy, data delivery to the base station, number of rounds and live nodes.
\end{abstract}

Index Terms:WSNs, energy consumption, LEACH, LEACH-C, LEACH-1R, HEED.

\section{Introduction}

The need for information and the rapid development of microelectronics, micromechanics, and wireless technologies allowed to producing smaller sensor components with a low price for monitoring the physical quantities (heat, humidity, and vibrations) [1]. Recently, these components are provided with the communications system to communicate with the other sensors, which give birth to Wireless Sensor Networks (WSN). The Wireless Sensor Networks are different from the other wireless networks because they generally have the following characteristics: high density, low flow, low energy capacity, and an inaccessible environment [2]. The latter two characteristics have made energy as a major constraint since the sensor batteries are generally not rechargeable. To extend the lifetime of a wireless sensor network while ensuring the main tasks of a sensor node (capture, processing, and sending of data) the energy of sensor nodes must be conserved. Among these three tasks is sending data or communication is the task which consumes much energy. That is why researches are motivated to focus on the MAC layer (Medium Access Control) and network layer.

A wireless sensor network (WSN) in its simplest form can be defined as a network of (possibly low size and low-complex) devices denoted as nodes that can sense the environment and communicate the information gathered from the monitored field through wireless links [3]; the data is forwarded, possibly via multiple hops relaying, to a sink that can use it locally, or is connected to other networks (e.g., the Internet) through a gateway [4]. The set of challenges in sensor networks are diverse, but the power consumption is a central design consideration whether they are powered using batteries, however, security also is a very important and big challenge in WSNs. Energy is the biggest constraint to wireless sensor capabilities. It is assumed that once sensor nodes are deployed in a sensor network, they cannot be easily replaced (high operating cost) or recharged (high cost of sensors). Therefore, the battery charge taken with them to the field must be conserved to extend the life of the individual sensor node and the entire sensor network [5]. Moreover, sensors have limited computing power [6] and limited storage capacity. Sensor nodes generally include three types of memory: RAM, program memory and working memory. RAM is used to store temporary data when working, usually no more than 2kbytes; program memory for storing the operating system, applications, and security functions; and sensor memory which is used to store information.

Sensors are also characterized by limited communication range: the time of signal transmission for energy consumption, sensor nodes in the transmission of RF power module is generally between $10 \mathrm{~mW}$ to 100 MW, the transmission range is also limited to 100 meters to within $1 \mathrm{~km}$. For these reasons, many routing protocols were specifically developed to cope with limitations of sensor networks.The rest of this paper is organized as follows. Section II presents the general classification of routing protocols deployed in WSNs. In Sections III and IV, routing protocols (LEACH-like and HEED-like) are described for comparison purposes. The simulation results and analysis are presented in Section V. Section VI is the conclusion.

\section{Routing Protocols In Wireless Sensor Networks}

Routing protocols in WSNs are for settings up one or more path(s) from sensor nodes have limited resources, routing protocols should have small overhead which may result from control message interchange and caching [7]. So a good routing protocol must route data with low latency, consuming little energy, and minimizing load network and be reliable. 
Wireless Routing protocols are classified into many categories:

\subsection{Flat Routing Protocols}

In this category, each node plays the same role and sensor nodes collaborate to perform the sensing task [8]. Examples of flat routing protocols include: Sensor Protocols for Information via Negotiation (SPIN), Directed Diffusion, Rumor Routing, Gradient-Based Routing (GBR), Minimum Cost Forwarding Algorithm (MCFA), Constrained Anisotropic Diffusion Routing (CADR) and ACtive Query forwarding InsensoRnEtworks (ACQUIRE).

\subsection{Hierarchical Routing Protocols}

In these protocols, higher-energy nodes are used to process and send the information, while low-energy nodes are used to perform the sensing in the proximity of the target [9]. The creation of clusters and assigning special tasks to cluster heads can greatly contribute to overall system scalability, lifetime, and energy efficiency. Hierarchical routing is an efficient way to lower energy consumption within a cluster, performing data aggregation and fusion in order to decrease the number of transmitted messages to the sink node. Examples of hierarchical routing protocols include: Low Energy Adaptive Clustering Hierarchy (LEACH), Threshold sensitive Energy Efficient sensor Network (TEEN), AdaPtive Threshold sensitive Energy Efficient sensor Network Protocol (APTEEN), Power Efficient Gathering in Sensor Information Systems (PEGASIS), Hybrid Energy Efficient Distributed Protocol (HEED) and Stable Election Protocol (SEP).

\subsection{Location-Based Routing Protocols}

In this category, sensor nodes are addressed by means of their locations [8]. The distance between neighboring nodes can be estimated on the basis of incoming signal strengths. Relative coordinates of neighboring nodes can be obtained by exchanging such information between neighbors or by communicating with a satellite using GPS. To save energy, some location-based schemes demand that nodes should go to sleep if there is no activity. As examples of location-based protocols: Geographic Adaptive Fidelity (GAF), Geographic and Energy Aware Routing (GEAR) and Greedy Other Adaptive Face Routing (GOAFR).

\section{LEACH-Like Protocols}

\subsection{Low Energy Adaptive Clustering Hierarchy Protocol (LEACH)}

Low Energy Adaptive Clustering Hierarchy (LEACH) proposed by Wendi B. Heinzelman, et al. [10] is the first hierarchical, self-organizing, adaptive cluster-based routing protocol for wireless sensor networks which partitions the nodes into clusters. LEACH is a hierarchical protocol in which most nodes transmits the data to cluster heads, and the cluster heads aggregate and compress the data and forward it to the base station. Node first senses its target and then sends the relevant information to its cluster head. Then the cluster head aggregates and compresses the information received from all the nodes and sends it to the base station. Nodes that have been cluster heads cannot become cluster heads again for $\mathrm{P}$ rounds, where $\mathrm{P}$ is the desired percentage of cluster heads. Thereafter, each node has a 1/P probability of becoming a cluster head in each round. At the end of each round, each node that is not a cluster head selects the closest cluster head and joins that cluster. The cluster head then creates a schedule for each node in its cluster to transmit its data. Each sensor node $n$ generates a random number $r$ such that $0<r<1$ and compares it to a pre-defined threshold $T(n)$. If $r<T(n)$, the sensor node becomes cluster head in that round, otherwise it is cluster member.

After the cluster heads are selected, the cluster heads advertise to all sensor nodes in them network that they are the new cluster heads. Then, the other nodes organize themselves into local clusters by choosing the most appropriate cluster head (normally the closest cluster head). During the steady-state phase the cluster heads receive sensed data from cluster members, and transfer the aggregated data to the BS [11].

\subsection{Low Energy Adaptive Clustering Hierarchy Centralized Protocol (LEACH-C)}

LEACH-C uses a centralized clustering algorithm and the same steady-state phase as LEACH [12]. LEACH-C protocol can produce better performance by dispersing the cluster heads throughout the network [13]. During the set-up phase of LEACH-C, each node sends information about its current location (possibly determined using GPS) and residual energy level to the sink. In addition to determining good clusters, the sink needs to ensure that the energy load is evenly distributed among all the nodes. To do this, sink computes the average node energy, and determines which nodes have energy below this average.

Once the cluster heads and associated clusters are found, the sink broadcasts a message that obtains the cluster head ID for each node. If a cluster head ID matches its own ID, the node is a cluster head; otherwise the node determines its TDMA slot for data transmission and goes sleep until it's time to transmit data. The steadystate phase of LEACH-C is identical to that of the LEACH protocol [13]. 


\subsection{LEACH One-Round Protocol (LEACH-1R)}

In order to achieve the goal of enhancing the WSNs clustering, that need to perform more control of the clustering process, i.e., detecting $\mathrm{CHs}$ and their members [14]. In fact, such operation is based on two phases. In the first phase, $\mathrm{CHs}$ are selected upon the first round of LEACH mechanism. LEACH-1R can use any of the first rounds since the percentage $\mathrm{P}$ is more or less respected. In the second phase, the clusters are preserved and a new $\mathrm{CH}$ is selected only if the current one ran out of energy, (i.e., the battery level beyond certain threshold). In this case, a new $\mathrm{CH}$ is selected among the cluster members only taking in consideration the strength of the last received signal.

\section{HEED Like Protocols}

The overarching goal of Hybrid Energy Efficient Distributed Clustering Protocol (HEED) is to prolong network lifetime [15]. For this reason, cluster head selection is primarily based on the residual energy of each node. Measuring this residual energy is not necessary since the energy consumed per bit for sensing, processing, and communication is typically known and, hence, residual energy can be estimated.

The primary clustering parameter is used to probabilistically select an initial set of cluster heads, and the secondary parameter to "break ties" among them. The cluster range or radius is determined by the transmission power level used for intracluster announcements and during clustering. The cluster power level should be set to one of the lower power levels of a node, to increase spatial reuse, and reserve higher power levels for intercluster communication. These higher power levels should cover at least two or more cluster diameters to guarantee that the resulting intercluster overlay will be connected.

The secondary clustering parameter, intracluster communication cost, is a function of:

1) Cluster properties, such as cluster size.

2) Whether or not variable power levels are permissible for intracluster communication.

If the power level used for intracluster communication is fixed for all nodes, then the cost can be proportional to:

1) Node degree, if the requirement is to distribute load among cluster heads.

2) $1 /$ node degree, if the requirement is to create dense clusters. This means that a node joins the cluster head with minimum degree to distribute cluster head load (possibly at the expense of increased interference and reduced spatial reuse), or joins the one with maximum degree to create dense clusters [15].

\subsection{HEED-MIN and HEED-MAX}

We use the terms minimum degree cost and maximum degree cost to denote these cost types. Observe that intercluster communication is not incorporated in the cost function since local information is insufficient in this case.

\subsection{HEED-AMRP}

Now, consider the case when variable power levels are allowed for intracluster communication. Let MinPwr denote the minimum power level required by a node $v_{i}, 1 \leq i \leq M$, to communicate with a cluster head $u$, where $\mathrm{M}$ is the number of nodes within the cluster range. We define the average minimum reachability power (AMRP) as the mean of the minimum power levels required by all $\mathrm{M}$ nodes within the cluster range to reach $u$, i.e.,

AMRP $=\frac{\sum_{i=1}^{M} M_{i n P w r}}{M}$

If each node is allowed to select the appropriate power level to reach its cluster head, then AMRP provides a good estimate of the communication cost.

In addition, HEED distribution of energy consumption extends the lifetime of all the nodes in the network, which adds to the stability of the neighbor set. Nodes also automatically update their neighbor sets in multihop networks by periodically sending and receiving heartbeat messages [15].

\section{Simulation, Results and Analysis}

The implementation and simulation of routing protocols is the most important phase of our study, because it enables us to know which protocol has the best performance in terms of different metrics. In the literature, we found many simulation environments and network simulators that are available for network performance measurement. In our study, we selected the MATLAB environment because it very simple and has easy ways to create GUIs (Graphical User Interface).

The radio hardware dissipation model is a simple radio model. In this model, when the distance of a node transmitting data to other nodes or the base station is greater than the threshold, the multipath $\left(\varepsilon_{\mathrm{mp}}\right)$ fading channel model is used. When the distance between a node transmitting data to other nodes or the base station is less than threshold the free space $\left(\varepsilon_{\mathrm{fs}}\right)$ channel model is used (power loss). Thus, to transmit a L-bit message at distance $\mathrm{d}$, the radio transmission energy is given by:

$E_{T X}=\left\{\begin{array}{c}L * E_{\text {elec }}+L * \varepsilon_{f s} * d^{2}, \quad d<d_{0} \\ L * E_{\text {elec }}+L * \varepsilon_{m p} * d^{4}, \quad d \geq d_{0}\end{array}\right.$ 
- $\quad E_{\text {elec }}$ represents the energy consumption in the for sending or receiving one bit.

- $\quad \varepsilon_{\mathrm{fs}} * \mathrm{~d}^{2}$ and $\varepsilon_{\mathrm{mp}} * \mathrm{~d}^{4}$ is the amplifier energy that depends on the transmitter amplifier model.

- $\quad E_{\mathrm{da}}$ is data aggregation energy.

The energy consumption of receiving L-bit data is:

$-\quad \mathrm{E}_{\mathrm{RX}}(\mathrm{L})=\mathrm{L} * \mathrm{E}_{\text {elec }}$

\subsection{Hardware characteristics for MATLAB environment}

In our simulation, we use the same machine characteristics in all experimentations as shown in the table below.

Table 1. Hardware Characteristics

\begin{tabular}{ll}
\hline Hardware & Characteristics \\
\hline Processor & Intel(R) Core(TM) i3-2348M CPU @ 2.30GHz, 2300 MHz \\
Memory (RAM) & $4.00 \mathrm{Go}$ \\
Operation system & Microsoft Windows7 Professional 32bits \\
\hline
\end{tabular}

\subsection{Simulation Parameters}

In general, we need to specify the simulation area and the location of the base station, which are important parameters in any simulation. Some parameters are related to only few clustering methods like the transmission radius for HEEDs protocols.

Table 2. Parameters of 1st Set of Simulations

\begin{tabular}{|l|l|}
\hline Parameters & Values \\
\hline Number of nodes & 200 \\
\hline Location of BS & $(50,50)$ \\
\hline Simulation area & $100 \mathrm{~m} \times 100 \mathrm{~m}$ \\
\hline Node deployment & Random \\
\hline Packet size & 500 Bytes \\
\hline Initial energy & $0.1 \mathrm{~J}$ \\
\hline Eelec & $50 \mathrm{~nJ} / \mathrm{bit}$ \\
\hline Efs & $10 \mathrm{pJ} / \mathrm{bit} / \mathrm{m}^{2}$ \\
\hline Eda & $5 \mathrm{pJ} / \mathrm{bit} / \mathrm{sig}$ \\
\hline$P$ & $0.02,0.05,0.07,0.1$ \\
\hline Transmission radius & $10,20,30,40,50$ \\
\hline
\end{tabular}

\subsection{Results and Analysis}

\subsubsection{Variable values of $P$ and transmission radius}

Fig. 1 presents the number of rounds versus $\mathrm{P}$ for the three protocols LEACH, LEACH-C and LEACH1R. In general, when P increases the number of rounds decreases for LEACH and LEACH-C unlike LEACH$1 \mathrm{R}$. This is due to the fact that in LEACH-1R, when the number of clusters increases the members of each cluster decreases which make the cluster-heads consumes lesser energy.

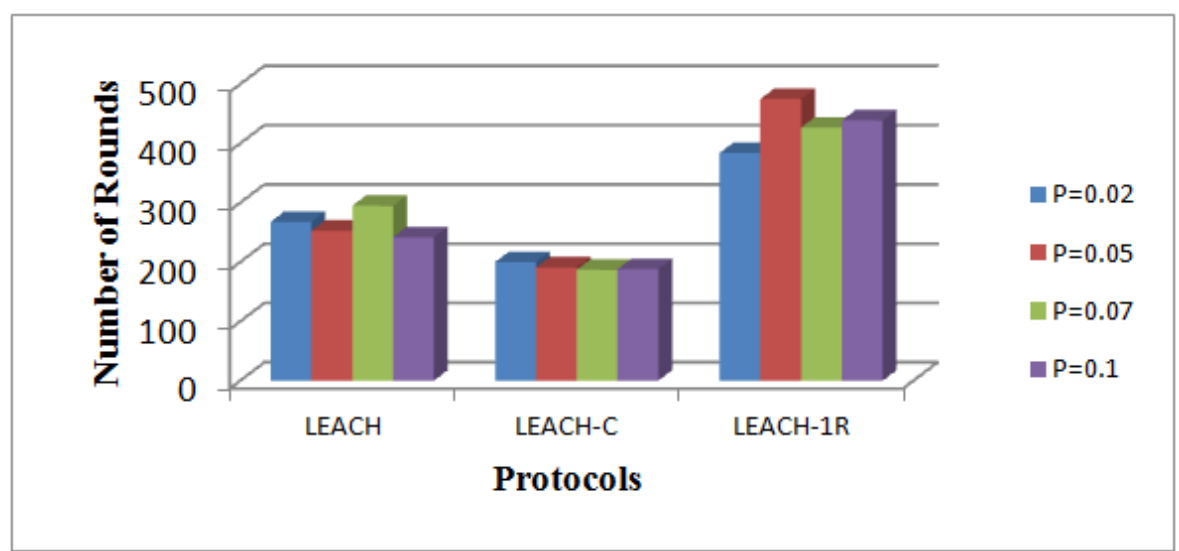

Fig. 1. Comparison of the number of rounds at different values of "P"

Fig. 2 presents the number of rounds versus the values of transmission radius for the HEEDs protocols. It can be clearly seen that the number of rounds decreases when increasing the transmission radius. This is due to the fact that increasing the radius results in more sensor nodes joining the same cluster, and therefore, the distance of sensor nodes to the cluster-head increases especially at the edges. Thus increasing the transmission radius affects the lifetime of networks. 


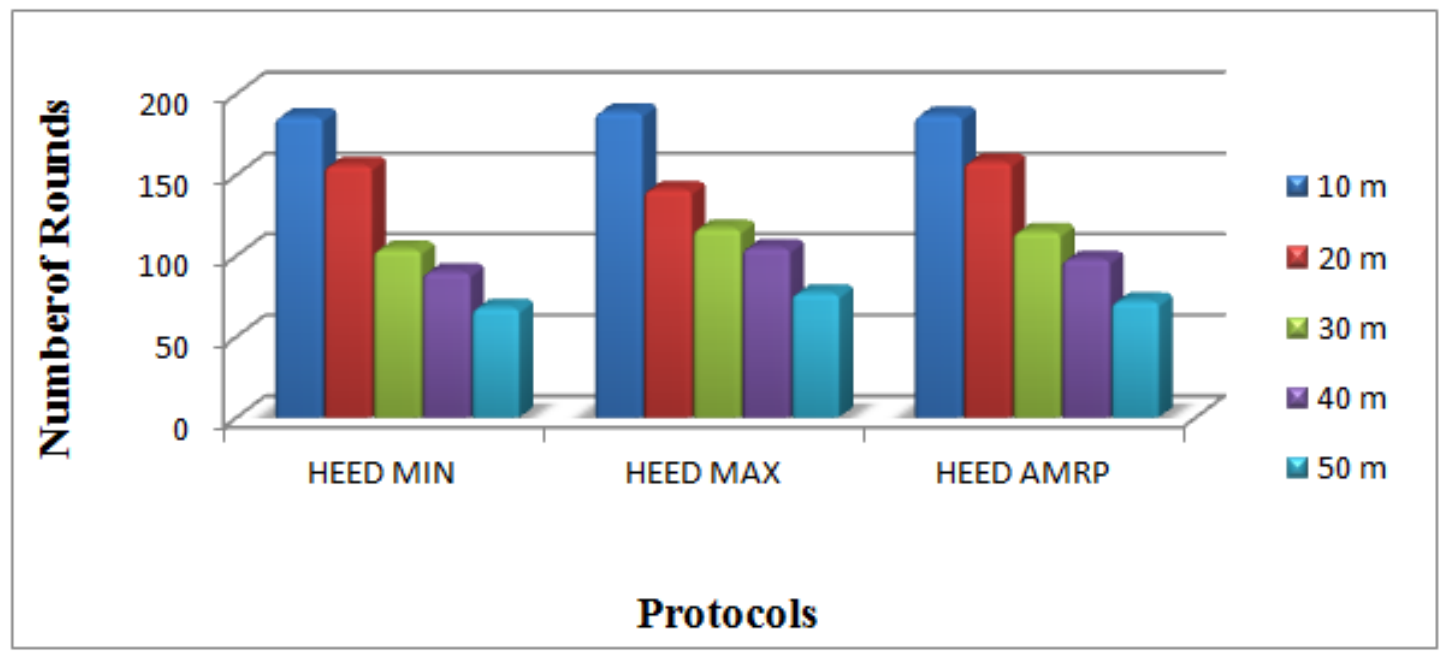

Fig. 2. Comparison of the number of rounds at different values of transmission radius

\subsubsection{The first scenario: $B S$ at the center}

Next, we fixed the values of $\mathrm{P}$ and the transmission radius to be 0.1 and $17 \mathrm{~m}$ respectively. These two values guarantee the creation of about the same number of clusters for all protocols (about 20 clusters). We also considered varying the position of the base station to test its impact on the selected protocols.

Table 3. Parameters of 2nd Set of Simulations

\begin{tabular}{|l|l|}
\hline Parameters & Values \\
\hline Number of nodes & 200 \\
\hline Location of BS & $(50,50)$ and $(50,175)$ \\
\hline Simulation area & $100 \mathrm{~m} \times 100 \mathrm{~m}$ \\
\hline Node deployment & Random \\
\hline Packet size & 500 Bytes \\
\hline Initial energy & $0.1 \mathrm{~J}$ \\
\hline Eelec & $50 \mathrm{~nJ} / \mathrm{bit}$ \\
\hline Efs & $10 \mathrm{pJ} / \mathrm{bit} / \mathrm{m}^{2}$ \\
\hline Eda & $5 \mathrm{pJ} / \mathrm{bit} / \mathrm{sig}$ \\
\hline Transmission radius & $17 \mathrm{~m}$ \\
\hline$P$ & 0.1 \\
\hline
\end{tabular}

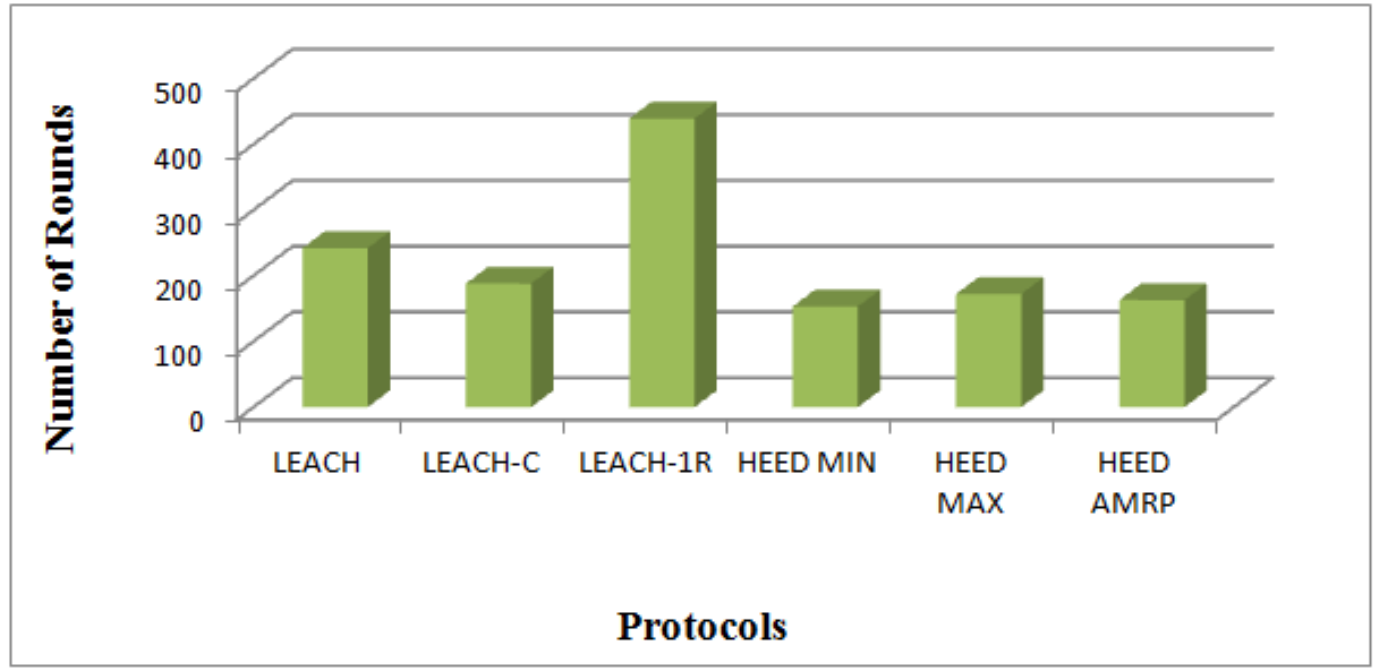

Fig. 3. Comparison of the lifetime of each protocol in the first scenario

Fig. 3 presents the number of rounds of each protocol when the $\mathrm{p}=0.1$, radius $=17 \mathrm{~m}$ and the location of base station is $(50,50)$. We observed that LEACH-1R has higher number of rounds, which is about 439 rounds, when compared to the others protocols. On the other hand, LEACH protocol has more lifetime than LEACH-C and HEEDs protocols. 


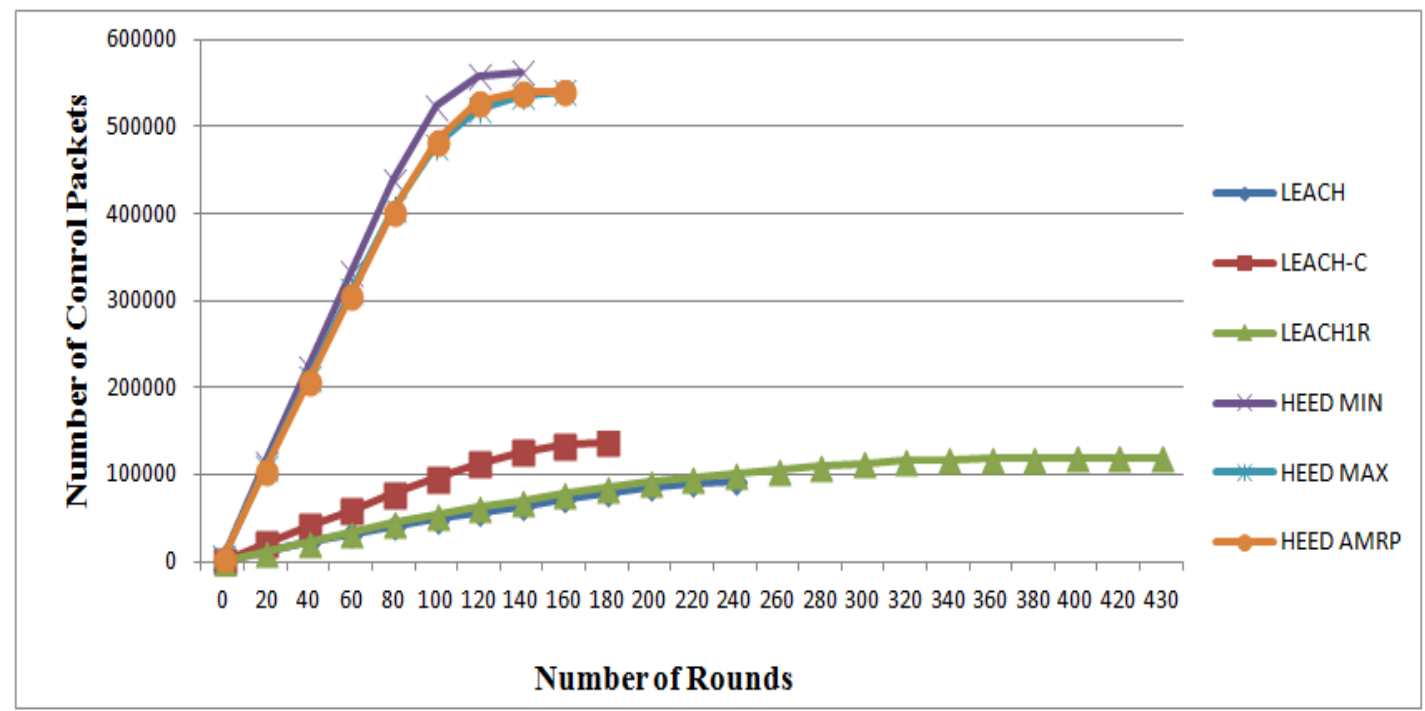

Fig. 4. Comparison of No. of control packets versus No. of rounds in the first scenario

Fig. 4 presents the number of control packets versus the number of rounds for the six protocols. It is clearly seen that the HEEDs protocols have more control packets because they suffer from a consequent overhead, since they need several iterations to find cluster heads (tentative $\mathrm{CH}$ and final $\mathrm{CH}$ ). Every iteration, a lot of control packets are broadcast. However, LEACH-C has more control packets when compared to LEACH and LEACH-1R because each node sends information about its current location and residual energy level to the BS. Then, the BS chooses the best $\mathrm{CH}$ and broadcasts a message that obtains the cluster head ID for each node which leads more control packets.

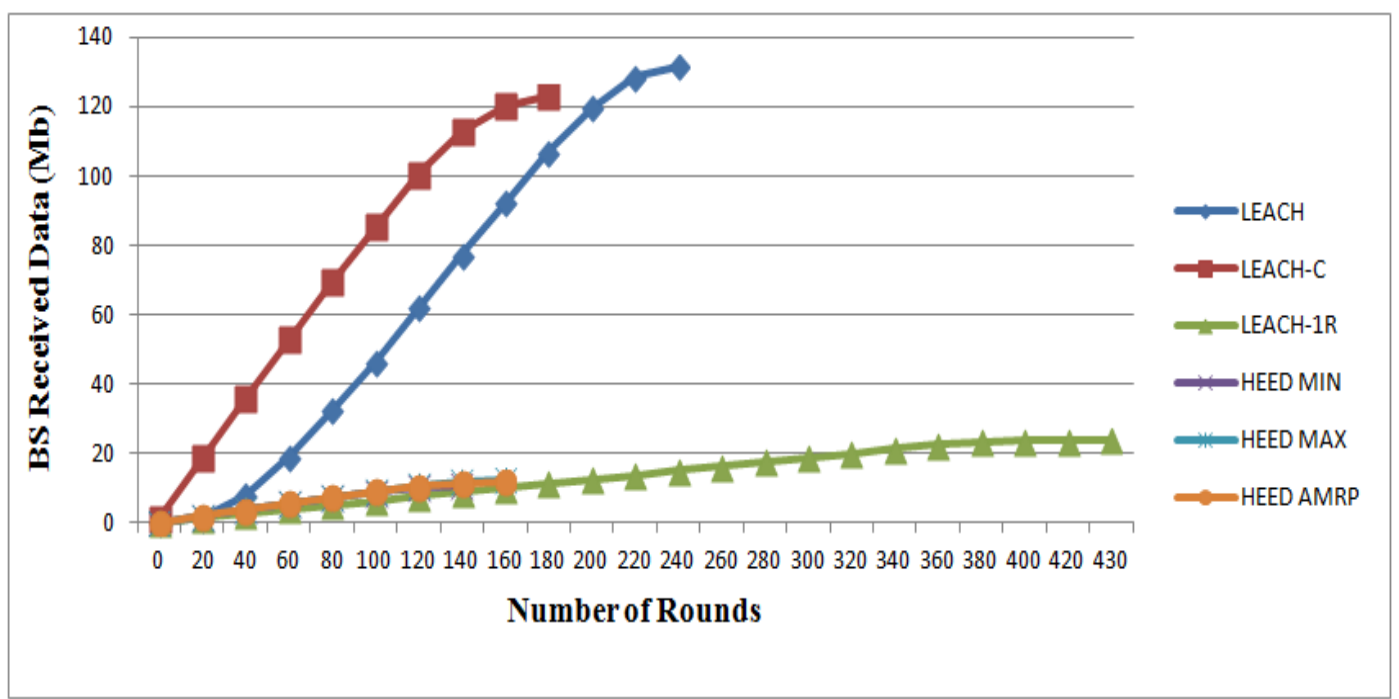

Fig. 5. Comparison of the data received by BS versus No. of rounds in the first scenario.

Fig. 5 presents the amount of data received by the base station versus the number of rounds for the six protocols. The simulations show that $\mathrm{LEACH}$ and $\mathrm{LEACH}-\mathrm{C}$ have more data delivery to BS, especially LEACH-C which uses network topology information to form good clusters, and then deliver more data to the sink. However, LEACH-1R and HEEDs protocols deliver almost the same amount of data because in LEACH$1 \mathrm{R}$ usually use the clusters with the same members, so the same data delivery was recorded in each rounds. But the delivery of more data to the sink does not mean that this protocol is better because sensors collect the same physical quantities (heat, humidity, vibrations...).That is why, the LEACH-1R and HEEDs are better than LEACH and LEACH-C protocols. 


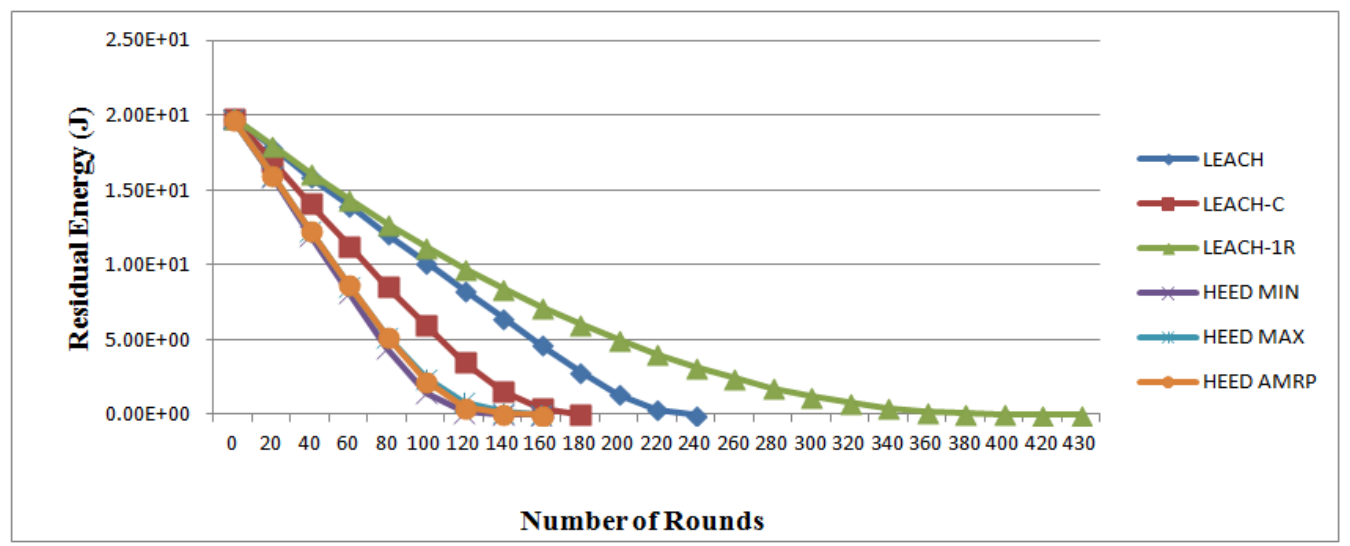

Fig. 6. Comparison of the residual energy versus No. of rounds in the first scenario

Fig. 6 presents the residual energy versus number of rounds for the six protocols. All protocols showed a gradual decrease of energy, but LEACH-1R has a regular decrease with the number of rounds until round 439 because it maintains the same members in each cluster and a new $\mathrm{CH}$ is selected only if the current one ran out of energy, unlike the other protocols which make sensors regularly change the clusters. This change leads to more control packets especially for HEEDs protocols, so more energy is consumed. When comparing LEACH and LEACH-C, results show that LEACH-C consumes more energy than LEACH; this is due the amount of data delivery to the $\mathrm{BS}$ and the large proportion of control packets (to find $\mathrm{CHs}$ ).

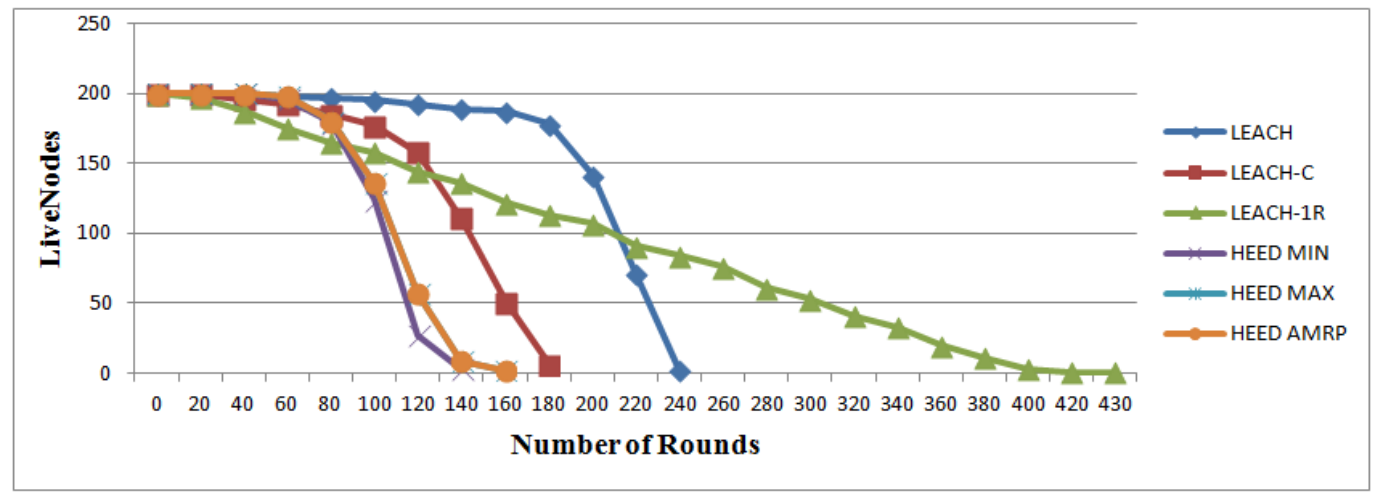

Fig. 7. Comparison of the live nodes versus No. of rounds in the first scenario

Fig. 7 presents the number of nodes that remain alive over the simulation round for the six protocols. We observed that after the first 20 rounds the number of live nodes for LEACH-1R protocols decrease because it does not changed the $\mathrm{CH}$ only after it runs out of energy. Due the mechanism of selection of $\mathrm{CHs}$ and the amount of data delivery, LEACH-C protocol shows an early decrease after 40 rounds compared to LEACH and HEEDs protocols. As illustrated in Fig. 7, the last $50 \%$ of the nodes started rapidly dying for all protocols except for LEACH-1R.

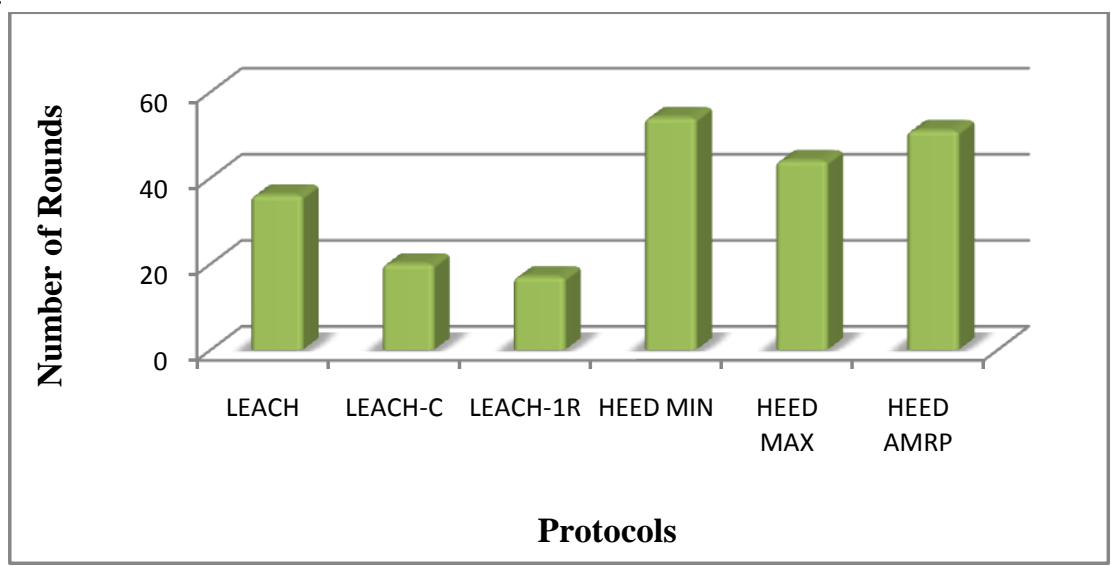

Fig. 8. The first dead node though the simulation rounds in the first scenario 
Fig. 8 presents the first dead node through the simulation rounds for the six protocols. It shows that LEACH-1R is the first protocol losing the first node because this protocol does not change the $\mathrm{CH}$ only after running out of energy, so the first dead node is the cluster head itself. However, HEEDs protocols are better 3 times than LEACH-1R due the distributed clustering method especially with HEED MIN protocol. The node chooses the cluster which has minimum members and joins it, so has no load in the cluster which conserves more energy in the first rounds. On the other hand, LEACH is better twice than LEACH-C relative to this metric because LEACH-C suffers more with control packets before choosing the $\mathrm{CH}$.

\subsubsection{The second scenario: Changing the BS location}

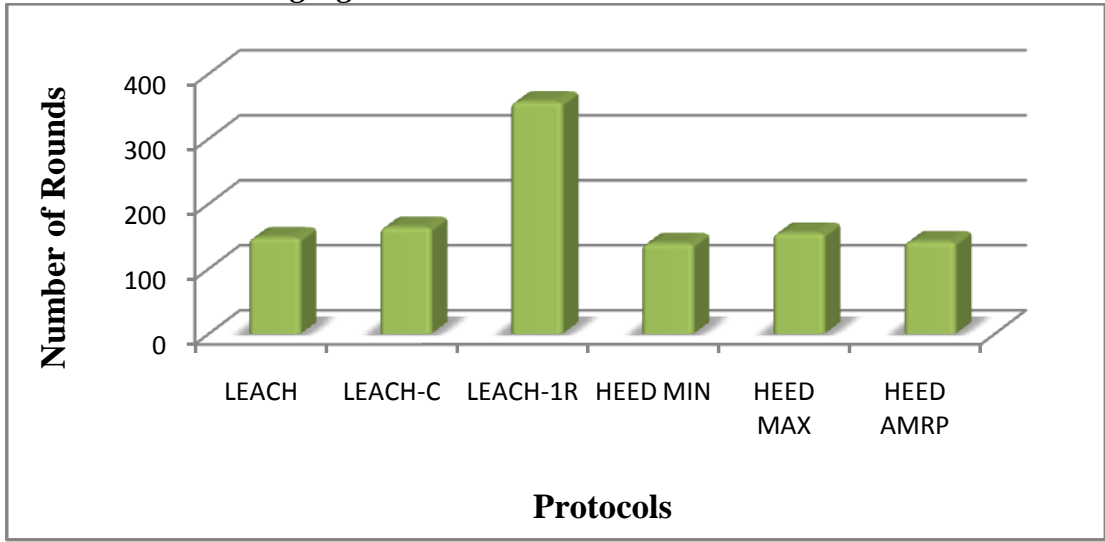

Fig. 9. Comparison of the lifetime of each protocol in second scenario

Fig. 9 presents the number of rounds of each protocols when the $\mathrm{p}=0.1$, radius $=17 \mathrm{~m}$ and the location of base station is at $(50,175)$, i.e., far from the simulation zone's center. Fig. 9 shows that the number of rounds for all protocols deceases as compared to the first scenario (BS at the center). Usually, LEACH-1R has higher number of rounds which is about 357 rounds as compared to the others protocols. However, LEACH-C and HEED MAX maintain almost the same life time compared to LEACH, HEED MIN and HEED AMPR protocols. This result is almost the contrary of what we found when the base station was located in the middle of networks.

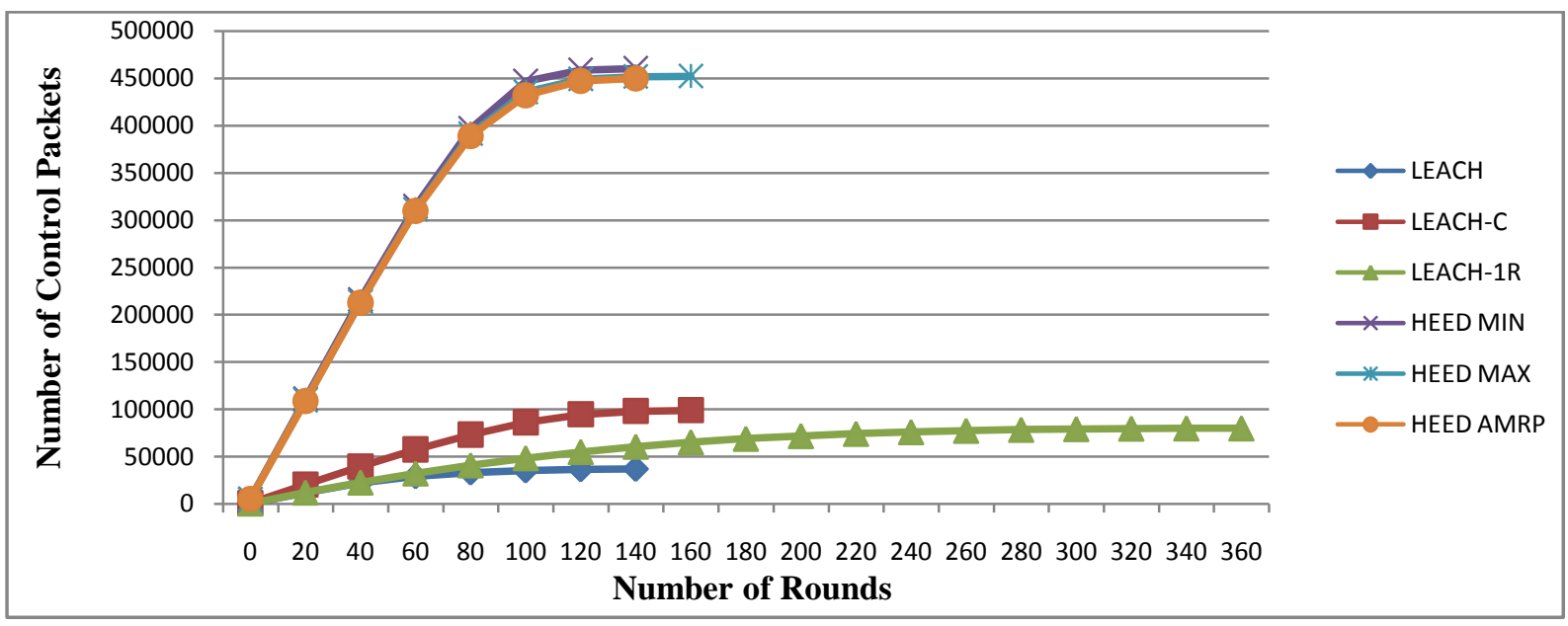

Fig. 10. Comparison of control packets of each protocol in the second scenario

Fig. 10 presents the number of control packets versus the number of rounds for the six protocols. We observed that the results are almost the same as the first scenario. 


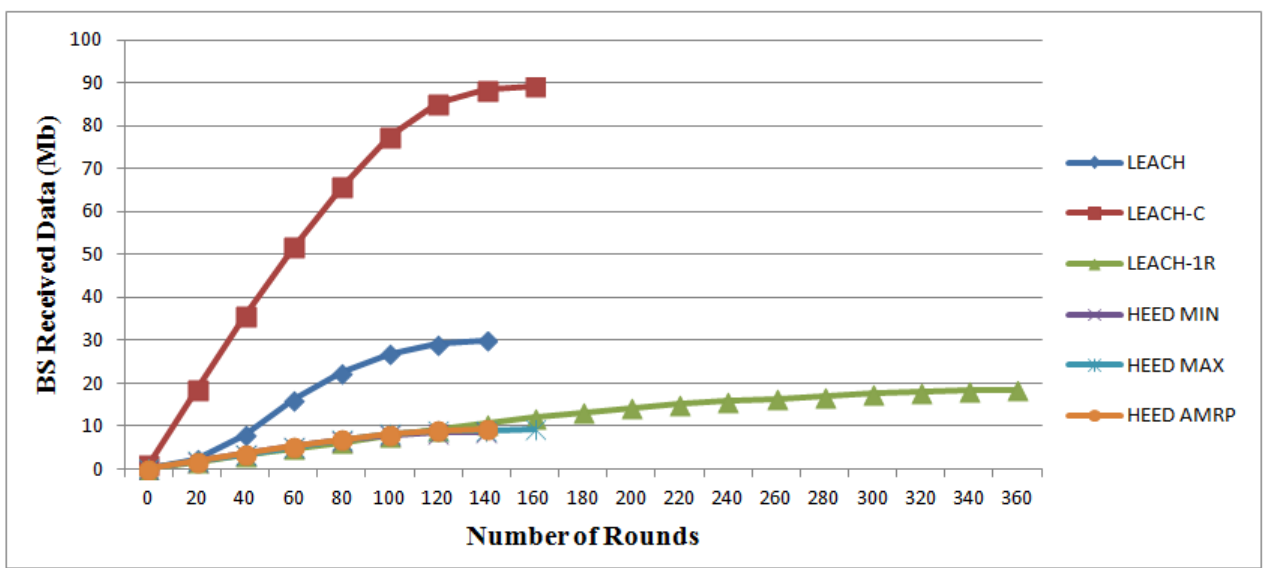

Fig. 11. Comparison of the data received by BS versus number of rounds in the second scenario

Fig. 11 presents the amount of data received by the base station versus the number of rounds for the six protocols. It shows that LEACH-C has more data delivery (3 times more) than LEACH because the BS is so far. In LEACH-C protocol the BS choose the CHs which allow it to delivery more data unlike LEACH. On the other hand, LEACH-1R and HEEDs protocols deliver almost the same amount of data.But when comparing LEACH$\mathrm{C}$ and LEACH for the two location of BS, we conclude that, these two protocols perform well when the BS is so far, which reduces the amount of data delivery to the BS.

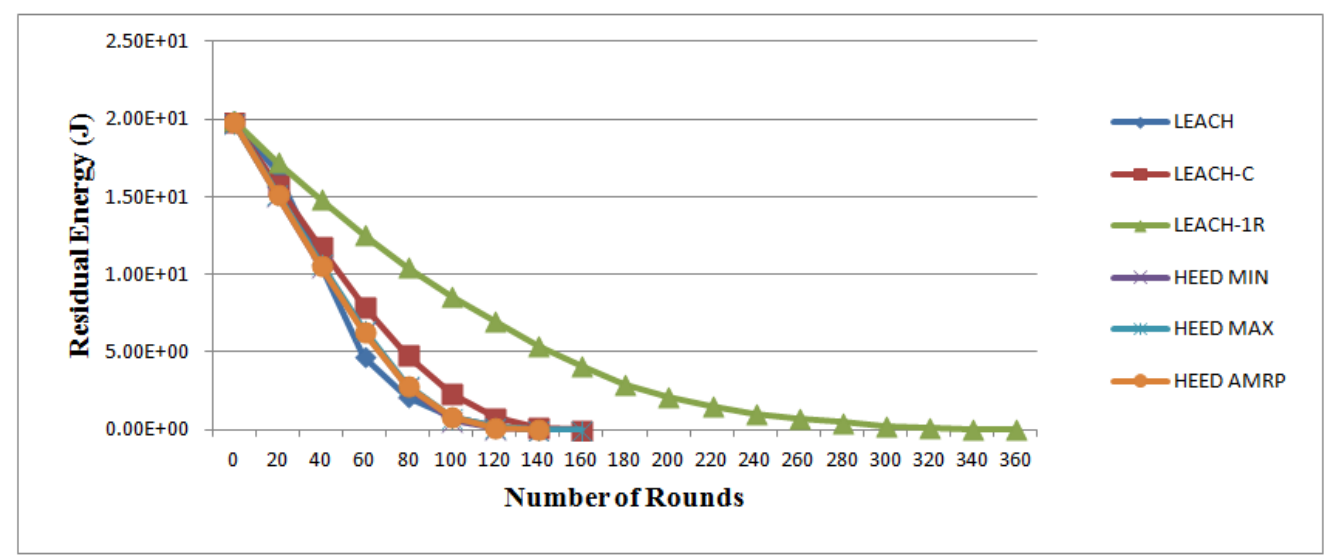

Fig. 12. Comparison of the residual energy No. of rounds in the second scenario

Fig. 12 presents the residual energy versus the number of rounds for the six protocols. All protocols showed a gradual decrease of energy and the difference between protocols is very small expect LEACH-1R because it has no change of clusters unlike the other protocols. When comparing LEACH and LEACH-C, the former consumes more energy than the latter due the position of the base station, the selection of $\mathrm{CHs}$, and the distance between sensors and the $\mathrm{CH}$. However, the HEEDs protocols have almost the same results with the first scenario.

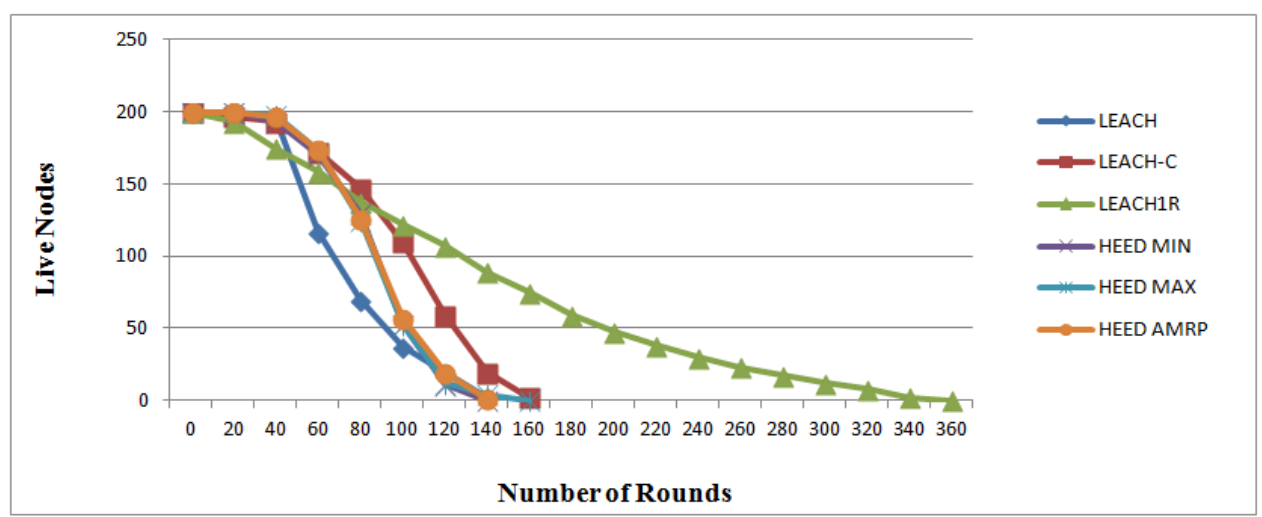

Fig. 13. Comparison of the live nodes versus No. of rounds in the second scenario 
Fig. 13 presents the number of nodes that remain alive over the simulation round for the six protocols. We observed in LEACH protocol the nodes die rapidly with the increase of the number of rounds because the $\mathrm{CHs}$ are not uniformly distributed within the cluster. Therefore, $\mathrm{CHs}$ can be located at the edges of the cluster, and the location of BS is so far which need more and more energy. In contrast, LEACH-C has regular decrease in the number of live nodes due the mechanism of selection of the CHs (Location and energy). For the HEEDs protocols, the decrease of live nodes is between that of LEACH and LEACH-C because the $\mathrm{CH}$ selection is primarily based on the residual energy of each node which is the best parameters especially when the BS is so far. For the LEACH-1R protocol, the live nodes decrease uniformly because it has no change of clusters and $\mathrm{CH}$ is changed only if it runs out of energy.

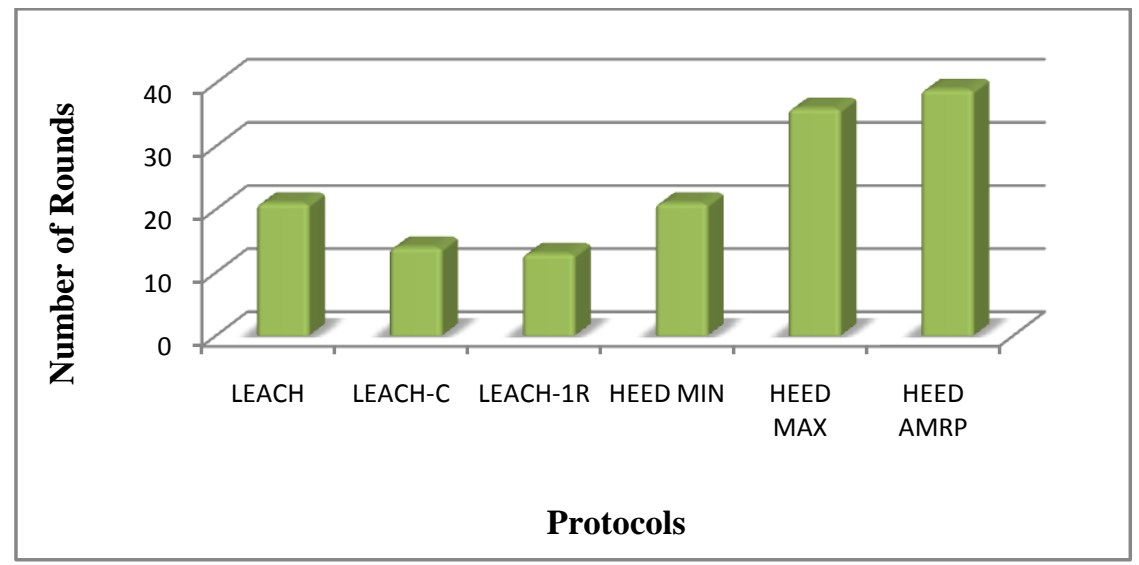

Fig. 14. The first node dies though the simulation rounds in the second scenario

Fig. 14 presents the first dead node though the simulation rounds for the six protocols. As shown in the first scenario, LEACH-1R is the first protocol losing the first node. So, LEACH-1R protocol has no relation with the position of BS in this metric. However, HEEDs protocols are better than others due to the distributed clustering method. On the other hand, LEACH is better than LEACH-C because LEACH-C suffers more when sending control packets before choosing the $\mathrm{CH}$ which might force the first node to exhaust more battery power. As compared with the results of the first scenario, we show that the three protocols LEACH, LEACH-C and LEACH-1R have the same results, but the HEEDs protocols are clearly affected by the BS position.

\section{Conclusion}

Wireless sensor networks are expanding and being more and more prevalent. Currently, they are very dynamic research area where enhancements are constantly being sought, adding to their applications are very numerous and diverse, but the major problem in sensor networks is the conservation of the energy.

In this paper, different routing protocols have been evaluated (LEACH, LEACH-C, LEACH-1R, HEED MIN, HEED MAX, and HEAD AMRP) in WSN. These routing protocols are compared using MATLAB environment on Windows7. The comparison is based on the number of control packets, number of rounds, live nodes, data delivery to the base station and the residual energy in each round. We conducted two different scenarios with different locations of the BS.

Performance of each protocol has been analyzed and evaluated in each scenario. Our simulation results indicate that LEACH has the best performance in the first scenario when the base station was in the middle of the area according to the performance metrics which have been selected. In other hand, LEACH-C works well in the second scenario on contrary with LEACH protocol, due the best selection of CHs. The rest of the protocols (HEEDs and LEACH-1R) are almost gave us the same performance in both of scenarios. In addition, LEACH-1R showed the best performance in terms of residual energy, live nodes, and data delivery to the BS.

Finally, for the future work may consider implementing other routing protocols in wireless sensor networks and adding other parameters and other metrics like scalability and ratio of dropped packets.

\section{References}

[1]. Cayirci, E., 2003. Data aggregation and dilution by modulus addressing in wireless sensor networks. IEEE Communications Letters, 7(8), pp.355-357.

[2]. Handy, M.J., Haase, M. and Timmermann, D., 2002. Low energy adaptive clustering hierarchy with deterministic cluster-head selection. In Mobile and Wireless Communications Network, 2002. 4th International Workshop on (pp. 368-372). IEEE.

[3]. Mahizh, P. and Muthuselvi, D.V.K., 2013. A Maximum Residual Energy for WSN Using a Novel A-Star Algorithm And Neuro Fuzzy Approach.International Journal on Current Issues of Computer Science, 1(1).

[4]. A. B. Pagi, V. R. Budyal, and M. J. Sataraddi, 2014. Fuzzy Based Energy Aware Flat Routing (FEAFR) in Wireless Sensor Networks. International Journal of Emerging Technology and Advanced Engineering. 
[5]. Akyildiz, I.F., Su, W., Sankarasubramaniam, Y. and Cayirci, E., 2002. A survey on sensor networks. IEEE communications magazine, 40(8), pp.102-114

[6]. Joby, P.P., 2015. A Survey on Threats and Security schemes in Wireless Sensor Networks. International Journal of engineering Research and Applications, 1(5), pp.89-94.

[7]. Al-Ma'aqbeh, F., Banimelhem, O., Taqieddin, E., Awad, F. and Mowafi, M., 2012. Fuzzy logic based energy efficient adaptive clustering protocol. In Proceedings of the 3rd International Conference on Information and Communication Systems (p. 21). ACM.

[8]. Nikolidakis, S.A. and Vergados, D.D., 2013. Energy-efficient routing protocols in wireless sensor networks: A survey. IEEE Communications Surveys \& Tutorials, 15(2), pp.551-591.

[9]. Kour, H., 2012. Hierarchical Routing Protocols In Wireless Sensor Networks.International Journal of Information Technology and Knowledge Management,6(1), pp.47-52.

[10]. Heinzelman, W.R., Chandrakasan, A. and Balakrishnan, H., 2000. Energy-efficient communication protocol for wireless microsensor networks. In System sciences, 2000. Proceedings of the 33rd annual Hawaii international conference on (pp. 10-pp). IEEE.

[11]. Himanshi, S.V. and Soni, M.K., 2013. Study of Wireless Sensor Network Using LEACH Protocol. International Journal of Innovative Technology and Exploring Engineering, ISSN, pp.2278-3075.

[12]. Heinzelman, W.B., Chandrakasan, A.P. and Balakrishnan, H., 2002. An application-specific protocol architecture for wireless microsensor networks.IEEE Transactions on wireless communications, 1(4), pp.660-670.

[13]. Panchal, S., Raval, G. and Pradhan, S.N., 2010. Optimization of Hierarchical Routing Protocol for Wireless Sensor Networks with Identical Clustering. In Proceedings of the 2010 International Conference on Advances in Communication, Network, and Computing (pp. 119-123). IEEE Computer Society.

[14]. Omari, M., 2014. LEACH-1R: Enhancing The LEACH Protocol Using The First Round Clusters. International Journal of Computer and Information Technology, 3(5), pp.1403-1408.

[15]. Younis, O. and Fahmy, S., 2004. HEED: a hybrid, energy-efficient, distributed clustering approach for ad hoc sensor networks. IEEE Transactions on mobile computing, 3(4), pp.366-379. 